\title{
Adaptive Filterbank Multicarrier Wireless Systems for Indoor Environments
}

\author{
Alexander M. Wyglinski Peter Kabal Fabrice Labeau \\ Department of Electrical \& Computer Engineering \\ McGill University, Montréal, Canada H3A 2A7 \\ \{alexw, kabal, flabeau\}@TSP.ECE.MCGill.CA
}

\begin{abstract}
We investigate the use of modulated filterbanks in adaptive wireless multicarrier systems operating in indoor environments. The motivation for using filterbanks, as opposed to OFDM, is that these spectrally selective modulation filters can decrease the amount of interchannel interference without using lengthy cyclic prefixes which are necessary in OFDM. The design of the synthesis and analysis filterbanks, based on a single lowpass prototype filter, is presented. Furthermore, optimal subcarrier MMSE equalization, adaptive bit and power loading, as well as null subcarrier placement techniques are employed to enhance system throughput and bit error rate performance when operating in frequency selective channels. The performance of this system is studied and compared with an IEEE 802.11a-compliant system, which is based upon OFDM modulation.
\end{abstract}

\section{INTRODUCTION}

Multicarrier modulation (MCM) systems have been extensively used in high-speed data transmission applications. Their main characteristic over other modulation schemes is that they use multiple subcarriers to transmit data in parallel across the channel. As a result, MCM systems are capable of longer symbol periods for a given data rate, and this translates into reduced intersymbol interference (ISI) and improved system performance with respect to probability of error. One application where MCM systems are prevalent is wireless local area networks (WLANs), where data rates of up to 54 megabits per second $(\mathrm{Mb} / \mathrm{s})$ are currently being supported for systems operating in indoor environments [1].

An efficient version of MCM found in WLAN systems and other applications is orthogonal frequency division multiplexing (OFDM), which modulates and demodulates data onto orthogonal subcarriers using Inverse Discrete Fourier Transforms (IDFTs) and Discrete Fourier Transforms (DFTs). A cyclic prefix is used to mitigate the effects of ISI. Conventional OFDM systems, where the same type and size of signal constellation is applied to all subcarriers, form the crux of several WLAN standards, including IEEE 802.11a [1] and ETSI HIPERLAN/2 [2]. The primary drawback of conventional OFDM is that the overall error probability of the system is dominated by the subcarriers with the worst performance. Thus, to enhance system performance, these poorlyperforming subcarriers need to be suppressed. This is the rationale behind adaptive MCM.

Adaptive MCM improves system performance by distributing system resources to the subcarriers in such a way so as

This research was partially funded by the Natural Sciences and Engineering Research Council of Canada (NSERC) and Le Fonds de Recherche sur la Nature et les Technologies du Québec to minimize the overall error probability of the system. Two parameters that have been commonly adjusted on a per subcarrier basis are the transmission rates [3-6] (the signal constellation type and size of each subcarrier is determined by the channel conditions) and the transmit power levels [5] (such that larger signal constellations can be used per subcarrier). Most research has been focused on applying these schemes only to OFDM systems. In this paper, we investigate the performance of another type of MCM system, known as a filterbank multicarrier (FB-MC) system. In our implementation, both adaptive bit and power reallocation are employed to minimize the overall error probability of the system. Furthermore, the system is constrained to less than a maximum bit error rate, even at the cost of some data throughput. Finally, optimal minimum mean-squared error (MMSE) equalizers are applied to each subcarrier to further improve performance. The number of taps for each subcarrier equalizer varies based on the amount of distortion.

This paper is organized as follows. In Section II, the implementation of our adaptive FB-MC system is described, the system parameters are presented, and indoor channel model used in this work is outlined. The derivation of the optimal subcarrier MMSE equalizer is outlined in Section III. Section IV contains the details of the bit, power, and equalizer tap allocation schemes employed in this paper. The results of the adaptive FB-MC system, an adaptive OFDM system, and an IEEE 802.11a-compliant system are presented and a comparison is conducted based on overall throughput in Section V. Finally, a summary of this work and some conclusions are given in Section VI.

\section{SySTEM MODEL}

\section{A. Filterbank Multicarrier Modulation}

The proposed system setup is shown in Fig. 1. An MCM system divides the input symbol stream into $N$ parallel streams, each having a higher symbol period and lower bandwidth than the input [7]. Each of these parallel streams is then used to modulate a carrier using a basis function $g^{(k)}(n), 1 \leq k \leq N$, as shown in Fig. 1(a). The modulated streams are then summed together and transmitted across the channel, where they experience the effects of multipath propagation due to the indoor environment, and noise. In Fig. 1(b), the received signal, $r(n)$, is decomposed into the $N$ subcarriers using basis functions $f^{(k)}(n), 1 \leq k \leq N$. The subcarrier equalizers $w^{(k)}(n)$, $1 \leq k \leq N$, are then applied to each of the received parallel streams and the result is multiplexed together to form the received symbol stream.

Unlike OFDM systems, which use IDFT and DFT basis 


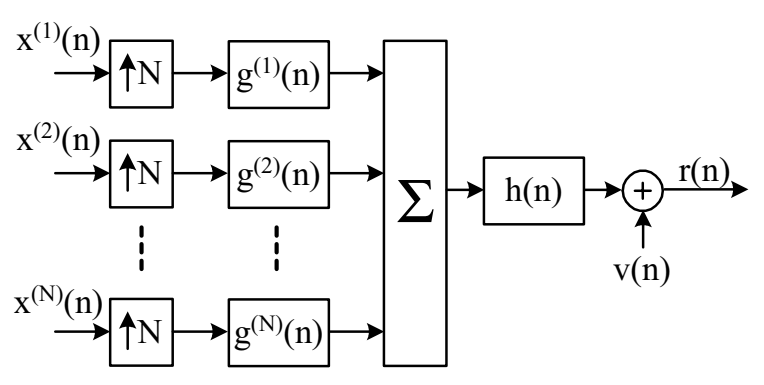

(a) transmitter and channel structures

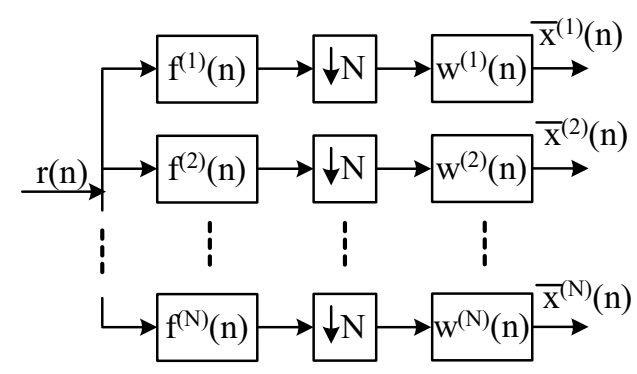

(b) receiver structure

Fig. 1 A schematic of the filterbank multicarrier data transmission system

functions $[7,8]$, our basis functions are based upon a modulated lowpass prototype filter, $p_{0}(n)$. The advantage of using these basis functions is that they can be designed to be more spectrally selective than IDFT and DFT basis functions, resulting in a MCM system with lower interference between subcarriers. To create the transmitter (or synthesis) filterbank and the receiver (or analysis) filterbank, the prototype filter $p_{0}(n)$, of length $P$, is modulated from $\omega=0$ rads to a center frequency $\omega_{k}$ using the expressions $[9,10]$

$$
g^{(k)}(n)=p_{0}(n) \exp \left[j \omega_{k}\left(n-\frac{P}{2}\right)+\theta_{k}\right]
$$

and

$$
f^{(k)}(n)=p_{0}(n) \exp \left[j \omega_{k}\left(n-\frac{P}{2}\right)-\theta_{k}\right] .
$$

Note that the filterbank has center frequencies

$$
\omega_{k}=\frac{\pi(2 k+1)}{N}
$$

and phase terms

$$
\theta_{k}=(-1)^{k-1} \frac{\pi}{4}
$$

where $1 \leq k \leq N$.

A square-root raised cosine filter was chosen for $p_{0}(n)$, although other filters could also be used. Its time domain representation is [11]

$$
p_{0}(n)=\frac{4 \alpha}{\pi \sqrt{T}} \frac{\cos ((1+\alpha) \pi n)+\sin ((1-\alpha) \pi n) / 4 \alpha n}{1-(4 \alpha n)^{2}}
$$

where $\alpha$ is the roll-off factor, which represents the normalized excess bandwidth occupied by the filter beyond its mini-
TABLE 1 SEVERAL OPERATING PARAMETERS

\begin{tabular}{lcl}
\hline \hline System Parameter & IEEE 802.11a & Adaptive FB-MC \\
\hline Data Rate $[\mathrm{Mb} / \mathrm{s}]$ & $6,9,12,24,36$, & $0-78$ \\
& 48,54 & \\
Mod. Scheme & BPSK, QPSK, & BPSK, QPSK, \\
& $16-\&$ 64-QAM & 16- \& 64-QAM \\
Coding Rates & 1/2, 2/3, 3/4 & N/A \\
No. of Subcarriers & $52(48 \mathrm{D}, 4 \mathrm{P})$ & 52 \\
Symb. Duration $[\mu \mathrm{s}]$ & $4(3.2 \mathrm{D}, 0.8 \mathrm{G})$ & 4 \\
\hline
\end{tabular}

mum bandwidth, and $T$ is the symbol period. The value of $\alpha$ is limited to the range $0 \leq \alpha \leq 1$. A useful property of squareroot raised cosine filters is that the overall magnitude response of the transmitter and receiver filters will be a Nyquist filter. Moreover, the overall phase response will be linear. Since the prototype filter was implemented as an FIR filter of length $P$, it will not be exactly a square-root raised cosine filter due to truncation. Nevertheless, matched filtering would be performed by the $k^{\text {th }}$ receiver and transmitter filters, thus maximizing the signal-to-noise ratio (SNR) of the received signal.

The choice of operating parameters, such as the number of subcarriers and modulation modes employed, will be discussed in the next subsection. The design of the subcarrier equalizer will be discussed in Section III.

\section{B. Operating Parameters}

To conduct a fair comparison with current WLAN standards, the adaptive FB-MC system used in this work has the same basic parameters found in IEEE 802.11a. The parameters for both systems are presented in Table 1.

Our adaptive FB-MC system uses 52 subcarriers, which can be individually modulated with BPSK, QPSK, rectangular 16QAM, or rectangular 64-QAM. Furthermore, null subcarriers can be inserted in any of these 52 subcarriers, and the composite symbol duration is $4 \mu \mathrm{s}$. To make the comparison straightforward, neither our system nor the IEEE 802.11a-compliant system used in the comparison employs convolutional encoding/decoding.

\section{Indoor Channel Model}

A channel impulse response that adequately modelled an indoor environment was required to evaluate the proposed system. Although several alternatives exist, such as physical measurements, ray tracing software, and statistical methods, it was decided that the indoor propagation modelling technique devised by Saleh and Valenzuela [12] be used.

In this technique, the transmissions are modelled as reflected clusters of rays whose reception at the receiver antenna can be mathematically described as a Poisson arrival process with mean arrival rate $\Lambda$. Furthermore, the rays in each cluster are also Poisson arrival processes with mean arrival rate $\lambda$. Finally, the amplitudes of the clusters and the individual rays both decrease exponentially with parameters $\Gamma$ and $\gamma$, respectively. Note that the parameters $\Lambda, \lambda, \Gamma$ and $\gamma$ are all dependent on the frequency and data rate of operation.

A sample frequency response of an indoor channel used in this work is shown in Fig. 2. It shows that the channel is fre- 
quency selective, with several deep nulls, and possesses a nonlinear phase response.
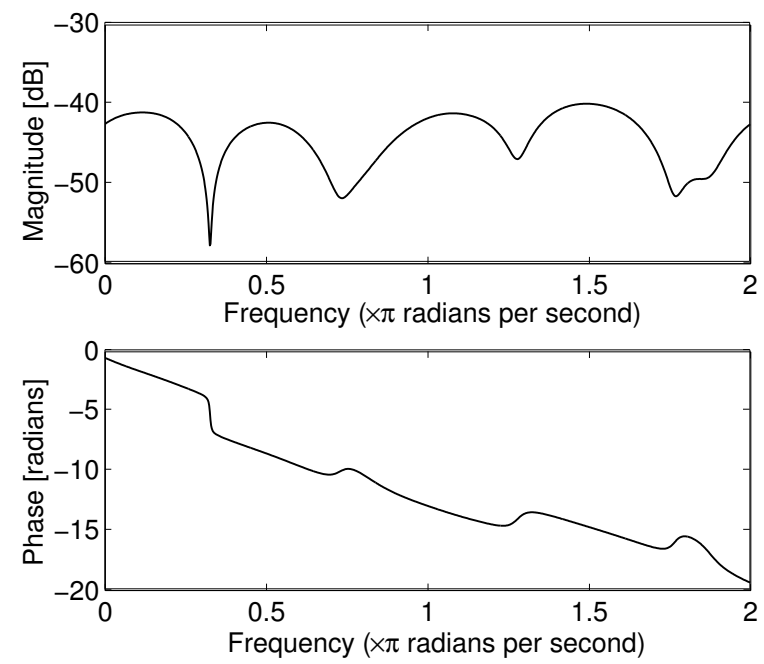

Fig. 2 Frequency response of an indoor channel environment in the 5.15-5.25 GHz U-NII band with transmitter/receiver distance of $50 \mathrm{~m}$

\section{OPTIMAL MMSE EQUALIZATION}

We saw in the previous section how the received signal was separated by the analysis filterbank into parallel streams. In addition to this process, the ISI distorted symbols need to be equalized in order to obtain the originally transmitted signal. Although zero-forcing (ZF) equalizers could be implemented, their performance is hampered by noise enhancement. Therefore, this work employs minimum mean-squared error (MMSE) equalizers. In the derivation that follows for the optimal MMSE equalizer, perfect channel knowledge is assumed. In reality, this information would be obtained through channel estimation techniques which use training sequences.

For the convenience of analysis, the data across each subcarrier is transmitted in finite blocks length. Thus, we define an input block of length $L$ transmitted across the $k^{\text {th }}$ subcarrier as

$$
\mathbf{x}_{n-L+1, n}^{(k)}=\left[\begin{array}{lll}
x^{(k)}(n) & \cdots & x^{(k)}(n-L+1)
\end{array}\right]^{T}
$$

where $\mathbf{x}^{T}$ is the transpose of $\mathbf{x}$.

The signal $\mathbf{x}_{n-L+1, n}^{(k)}$ is upsampled by $N$, filtered by the $k^{\text {th }}$ synthesis filter $\mathbf{g}_{n-P+1, n}^{(k)}$, a channel impulse response $\mathbf{h}_{n-S+1, n}$, and the $k^{\text {th }}$ analysis filter $\mathbf{f}_{n-P+1, n}^{(k)}$, before being downsampled by $N$ and equalized by $\mathbf{w}_{n-Q+1, n}^{(k)}$.

Filtering is performed in this analysis by using convolution matrices. Therefore, we can represent $\mathbf{g}_{n-P+1, n}^{(k)}$ as an $(N Q+$ $P+S-2) \times(N Q+2 P+S-3)$ convolution matrix

$$
\mathbf{G}^{(k)}=\left[\begin{array}{cccc}
\mathbf{g}_{n-P+1, n}^{(k) T} & 0 & \cdots & 0 \\
0 & \mathbf{g}_{n-P+1, n}^{(k) T} & \cdots & 0 \\
\vdots & \vdots & \ddots & \vdots \\
0 & 0 & \cdots & \mathbf{g}_{n-P+1, n}^{(k) T}
\end{array}\right] .
$$

Furthermore, the channel $\mathbf{h}_{n-S+1, n}$ and the $k^{\text {th }}$ analysis filter
$\mathbf{f}_{n-P+1, n}^{(k)}$, can be represented as $(N Q+P-1) \times(N Q+P+S-$ $2)$ and $(N Q) \times(N Q+P-1)$ convolution matrices, $\mathbf{H}$ and $\mathbf{F}^{(k)}$, respectively.

The upsampling and downsampling is performed using $(N Q+2 P+S-3) \times L$ and $Q \times(N Q)$ matrices $\mathbf{T}_{u}$ and $\mathbf{T}_{d}$, which are defined as

$$
\mathbf{T}_{u}=\left[\begin{array}{cccccccccccc}
0 & \cdots & 0 & 1 & 0 & \cdots & 0 & 0 & 0 & \cdots & 0 & 0 \\
0 & \cdots & 0 & 0 & 0 & \cdots & 0 & 1 & 0 & \cdots & 0 & 0 \\
\vdots & \ddots & \vdots & \vdots & \vdots & \ddots & \vdots & \vdots & \vdots & \ddots & \vdots & \vdots \\
0 & \cdots & 0 & 0 & 0 & \cdots & 0 & 0 & 0 & \cdots & 0 & 1
\end{array}\right]^{T}
$$

and

$$
\mathbf{T}_{d}=\mathbf{T}_{u}^{T}
$$

Therefore, the received signal prior to the equalizer is

$$
\hat{\mathbf{x}}_{n-Q+1, n}^{(k)}=\mathbf{T}_{d} \mathbf{F}^{(k)} \mathbf{H} \sum_{l=1}^{N} \mathbf{G}^{(l)} \mathbf{T}_{u} \mathbf{x}_{n-L+1, n}^{(l)}+\mathbf{T}_{d} \mathbf{F}^{(k)} \mathbf{v}_{n-S-P-N L+2, n}
$$

where $L$ was chosen to be

$$
L=\left\lfloor\frac{N Q+2 P+S-3}{N}\right\rfloor .
$$

Given Eq. (10), we apply the equalizer $\mathbf{w}_{n-Q+1, n}^{(k)}$ to it to get

$$
\bar{x}_{n}^{(k)}=\hat{\mathbf{x}}_{n-Q+1, n}^{(k) H} \mathbf{w}_{n-Q+1, n}^{(k)}
$$

where $\mathbf{x}^{H}$ represents the complex conjugate and transpose of the vector $\mathbf{x}$.

Therefore, using a mean squared error (MSE) cost function

$$
J^{(k)}=E\left\{\left|x_{n}^{(k)}-\bar{x}_{n}^{(k)}\right|^{2}\right\}
$$

we can obtain the MSE for the $k^{\text {th }}$ subcarrier as

$$
\begin{aligned}
J^{(k)} & =\sigma_{x, k}^{2}+\mathbf{w}_{n-Q+1, n}^{(k) H} \mathbf{R}_{x} \mathbf{w}_{n-Q+1, n}^{(k)}-\mathbf{w}_{n-Q+1, n}^{(k) H} \mathbf{p}_{x} \\
& -\mathbf{p}_{x}^{H} \mathbf{w}_{n-Q+1, n}^{(k)}+\mathbf{w}_{n-Q+1, n}^{(k) H} \mathbf{R}_{v} \mathbf{w}_{n-Q+1, n}^{(k)}
\end{aligned}
$$

where

$$
\begin{aligned}
\mathbf{R}_{x} & =\mathbf{T}_{d} \mathbf{F}^{(k)} \mathbf{H}\left(\sum_{l=1}^{N} \sigma_{x, l}^{2} \mathbf{G}^{(l)} \mathbf{T}_{u} \mathbf{T}_{u}^{H} \mathbf{G}^{(l) H}\right) \mathbf{H}^{\mathbf{H}} \mathbf{F}^{(k) H} \mathbf{T}_{d}^{H} \\
\mathbf{R}_{v} & =\sigma_{v}^{2} \mathbf{T}_{d} \mathbf{F}^{(k)} \mathbf{F}^{(k) H} \mathbf{T}_{d}^{H} \\
\mathbf{p}_{x} & =\sigma_{x, k}^{2} \mathbf{T}_{d} \mathbf{F}^{(k)} \mathbf{H} \mathbf{G}^{(k)} \mathbf{T}_{u}\left[\begin{array}{ll}
1 & \mathbf{0}_{1 \times(L-1)}
\end{array}\right]^{T}
\end{aligned}
$$

and $\sigma_{x, k}^{2}$ and $\sigma_{v}^{2}$ are the signal variance of subcarrier $k$ and the noise variance, respectively.

To get the minimum cost function, $J_{\min }^{(k)}$, we need to find the optimal MMSE equalizer weights. To do that, we differentiate Eq. (14) using [13]

$$
\frac{\partial}{\partial \mathbf{w}_{n-Q+1, n}^{(k) H}}=\frac{1}{2}\left[\begin{array}{c}
\frac{\partial}{\partial x_{0}}+j \frac{\partial}{\partial y_{0}} \\
\frac{\partial}{\partial x_{1}}+j \frac{\partial}{\partial y_{1}} \\
\vdots \\
\frac{\partial}{\partial x_{Q-1}}+j \frac{\partial}{\partial y_{Q-1}}
\end{array}\right]
$$

thus yielding, once we isolate $\mathbf{w}_{n-Q+1, n}^{(k)}$, the optimal equalizer weights to be

$$
\mathbf{w}_{n-Q+1, n}^{(k)}=\left(\mathbf{R}_{x}+\mathbf{R}_{v}\right)^{-1} \mathbf{p}_{x}
$$




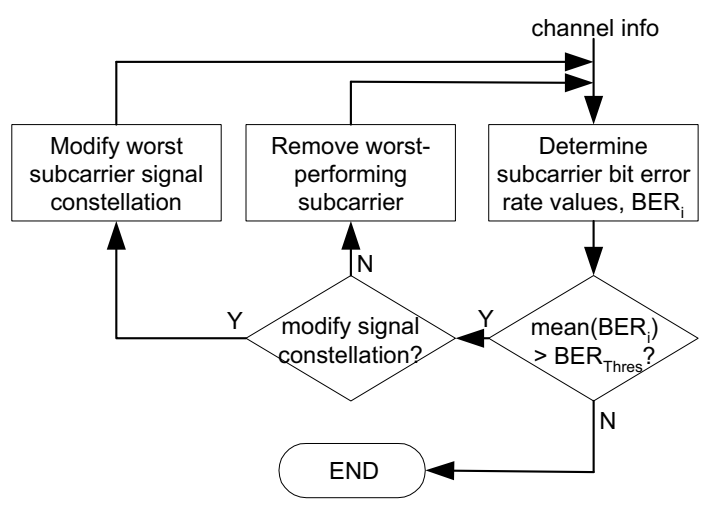

Fig. 3 Flow diagram of the adaptive bit reallocation algorithm

Now that the optimal MMSE equalizer has been determined, it can be used in conjunction with the algorithms of the next section to improve the performance of our system.

\section{Algorithm Descriptions}

\section{A. Bit Reallocation}

In this subsection, we will describe the bit loading algorithm used in this work. Assuming that all the signal constellations for the subcarriers are known, we use a greedy algorithm [14] to determine the optimal signal constellation configuration for the subcarriers given a specified target bit error rate (BER).

A flow diagram of the algorithm developed and used in this work is shown in Fig. 3. Initially, all the subcarriers are set to 64-QAM modulation. Having perfect knowledge of the subcarrier SNR per bit, $\gamma_{b}$, we use the probability of symbol error expression from [15] for M-ary QAM, namely

$$
\begin{aligned}
P_{M} & =4\left(1-\frac{1}{\sqrt{M}}\right) Q\left(\sqrt{\frac{3}{(M-1)} b \gamma_{b}}\right) \\
& \times\left[1-\left(1-\frac{1}{\sqrt{M}}\right) Q\left(\sqrt{\frac{3}{(M-1)} b \gamma_{b}}\right)\right],
\end{aligned}
$$

and use the approximation $P_{b} \approx P_{M} / b$, where $b$ is the number of bits per $M$-ary symbol ( $b$ is constrained to be even), to compute the BER for each subcarrier.

Once computed for all the subcarriers, the mean BER of the system is determined and compared against $B E R_{\text {Thres. If }}$ the mean BER is below $\mathrm{BER}_{\text {Thres, }}$, we keep this configuration. Otherwise, if the constraint is not satisfied, the non-nulled subcarrier with the worst BER is modified in order to improve the mean BER. If the signal constellation of the worst subcarrier is greater than BPSK, its size can be reduced and the entire process concerning the evaluation of the mean BER repeated to see if the $\mathrm{BER}_{\text {Thres }}$ constraint is satisfied. Otherwise, the worst subcarrier is nulled, the power is reallocated to the remaining non-nulled subcarriers if requested, and the process of determining the mean BER is repeated.

\section{B. Power Reallocation}

When subcarriers are nulled out, we have the option of recomputing the power levels of the non-nulled subcarriers in an attempt to support an increase in the number of levels. When subcarrier power reallocation is applied, the non-nulled sub- carrier $i$ has a power level of

$$
P_{i}=P_{1 \mathrm{MHz}}-\sum_{\substack{k=l \\ k \neq i}}^{l+2} P_{k}
$$

where $P_{1 \mathrm{MHz}}$ is the transmit power level constraint over any 1 $\mathrm{MHz}$ bandwidth. For the 5.15-5.25 GHz U-NII lower band [1], $P_{1 \mathrm{MHz}}$ is equal to $2.5 \mathrm{~mW}$. Since three consecutive subcarriers constitute $1 \mathrm{MHz}$, to determine $l$ we use

$$
\arg \max _{(i-2) \leq l \leq i}\left\{\sum_{k=l}^{l+2} P_{k}\right\}
$$

where the maximum power is constrained to be $P_{1 \mathrm{MHz}}$.

If all of the subcarriers are being used, each one has a transmit power level equal to $P_{1 \mathrm{MHz}} / 3$.

\section{Tap Allocation}

If the system has perfect channel knowledge, then a single optimal equalizer weight and minimum cost function can be determined for each subcarrier. If $J_{\mathrm{min}}^{(k)}$ exceeds a preestablished threshold for the subcarrier MSE, we repeat the process of determining the optimal equalizer weights and minimum cost function for subcarrier $k$ but we try for two taps instead. The process of adding equalizer taps is repeated until the MSE constraint is satisfied or until the difference between two successive cost functions is less than a specified percentage.

\section{Performance Results}

In the next two subsections, the resulting throughputs and tap allocations will be presented for adaptive OFDM and FBMC systems. Furthermore, the throughput of these systems will be compared with an IEEE 802.11a-compliant system. A $\mathrm{BER}_{\text {Thres }}$ of $10^{-3}$ was used in these experiments. Furthermore, the SNR is defined here as the nominal transmitted power divided by the noise power in the signal bandwidth. When measured this way, the SNR values tend to be large due to the channel attenuation.

\section{A. Throughput}

The throughput of an IEEE 802.11a-compliant system, an adaptive OFDM system, and our adaptive FB-MC system are shown in Fig. 4 for both 5 and 20 tap channel impulse responses.

It is observed in Fig. 4(a) that the adaptive OFDM and FBMC systems both exhibit similar throughput values across all SNR values. This is to be expected since both systems employ the optimal MMSE equalizer derived in Section III on a per subcarrier basis, the interchannel interference (ICI) caused by the channel is relatively small, and both systems are perfect or near-perfect reconstruction systems under ideal conditions. Moreover, because the channel impulse response is only 5 taps long, the IEEE 802.11a-compliant system is operational, although not at low SNR values.

In Fig. 4(b), the consequences of a long channel impulse response can be seen. In this case, the IEEE 802.11a-compliant system cannot satisfy the BER Thres constraint at any SNR due to the inability of the system's cyclic prefix to compensate for the ICI. Moreover, the adaptive OFDM system cannot reach 


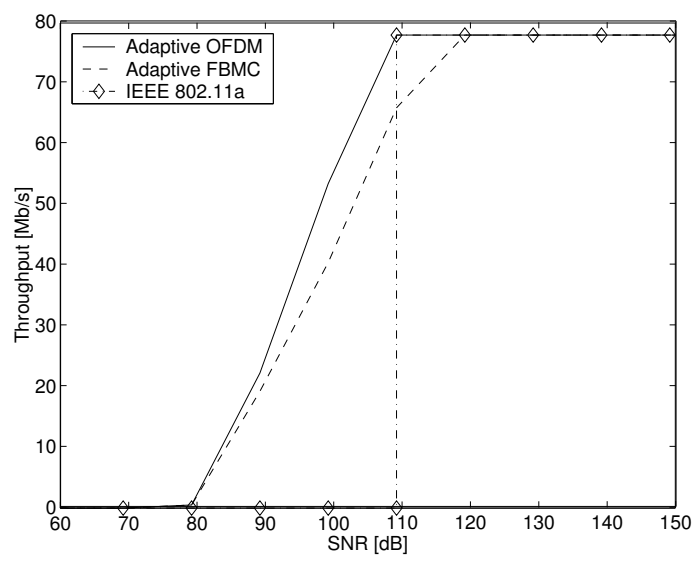

(a) 5-tap channel scenario

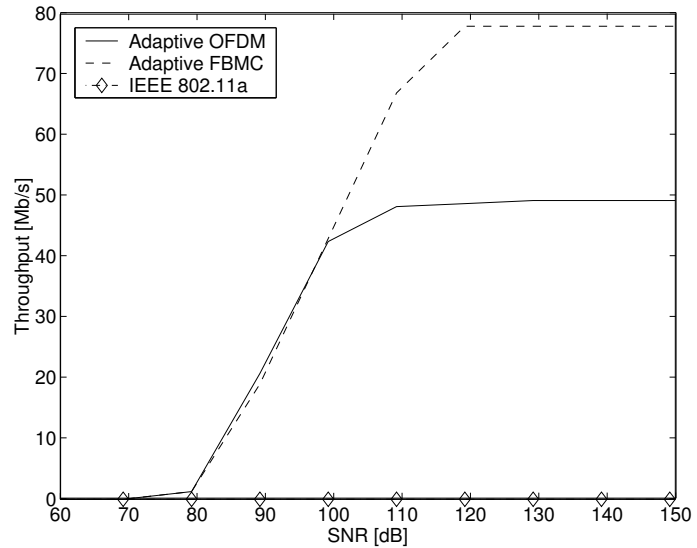

(b) 20-tap channel scenario

Fig. 4 Throughput of the adaptive OFDM, adaptive FB-MC, and IEEE 802.11a systems

the maximum throughput of $78 \mathrm{Mb} / \mathrm{s}$ due to the dominance of ICI in the cost function $J_{\min }^{(k)}$. On the other hand, the adaptive FB-MC system is still capable of achieving maximum throughput since it can better cope with the ICI.

\section{B. Equalizer Tap Allocation}

The total equalizer tap allocations for the adaptive OFDM and FB-MC systems are shown in Fig. 5. It is observed that when the SNR is low, very few subcarriers are used, therefore there are not many places where taps can be allocated in the first place. As the SNR increases, there are fewer nulled subcarriers and more taps can be assigned. As the noise power decreases even further, the system needs only a few taps to achieve adequate performance.

\section{CONCLUSION}

In this paper the throughput performance of an adaptive wireless multicarrier system which uses filterbanks and optimal subcarrier MMSE equalizers has been presented. The design process of the filterbank structure using a single lowpass prototype filter has been outlined. The derivation of the optimal MMSE equalizer specifically tailored for the adaptive FB-MC system has been described. Finally, the bit, power,

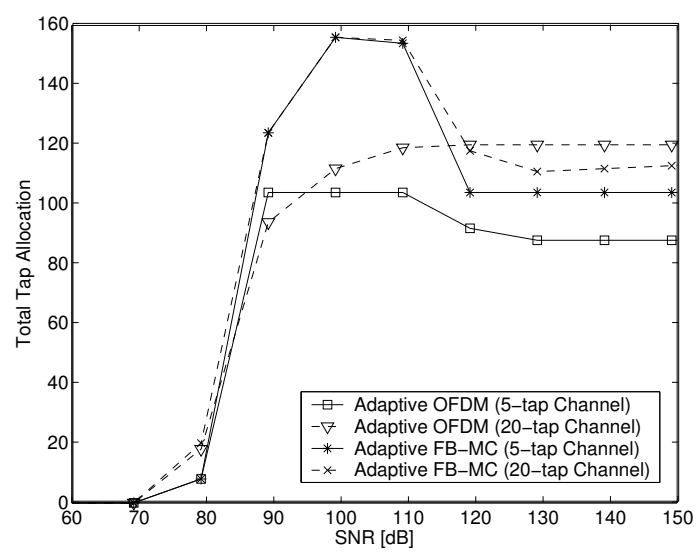

Fig. 5 Total tap allocations for the adaptive OFDM and FB-MC systems

and equalizer tap allocation schemes used by this system have been outlined. The results indicate that adaptive FB-MC systems exhibit improved performance relative to adaptive OFDM systems or an IEEE 802.11a-compliant system.

\section{ACKNOWLEDGMENTS}

The authors would like to thank Mr. Ricky Der with the verification of the derivations in Section III.

\section{REFERENCES}

[1] IEEE Std. 802.11a, "Wireless LAN medium access control (MAC) and physical layer (PHY) specifications: High-speed physical layer in the 5 GHz band," Nov. 1999.

[2] ETSI TS 101 475, "Broadband radio access networks (BRAN): HIPERLAN type 2; physical (PHY) layer," Dec. 2001.

[3] T. Keller and L. Hanzo, "A convenient framework for time-frequency processing in wireless communications," Proc. Inst. Elec. Electron. Eng., vol. 88, pp. 611-640, May 2000.

[4] A. Czylwik, "Adaptive OFDM for wideband radio channels," Proc. IEEE Global Telecommun. Conf., vol. 1, pp. 713-718, 1996.

[5] S.-T. Chung and A. Goldsmith, "Adaptive multicarrier modulation for wireless systems," Proc. Asilomar Conf. Signals, Systems and Computers, vol. 2, pp. 1603-1607, 2000.

[6] A. N. Barreto and S. Furrer, "Adaptive bit loading for wireless OFDM systems," Proc. IEEE Int. Symp. Personal, Indoor, Mobile Radio Commun., vol. 2, pp. 88-92, 2001.

[7] J. Louveaux, Filter Bank Based Multicarrier Modulation for xDSL Transmission. PhD thesis, Université Catholique de Louvain, Louvainla-Neuve, Belgium, May 2000.

[8] J. Heiskala and J. Terry, OFDM Wireless LANs: A Theoretical and Practical Guide. Sams Publishing, 2002.

[9] P. P. Vaidyanathan, Multirate Systems and Filter Banks. Prentice Hall Signal Processing Series, P T R Prentice Hall, 1993.

[10] R. P. Ramachandran, Bandwidth Efficient Filter Banks for Transmultiplexers. PhD thesis, McGill University, Montréal, Canada, September 1990.

[11] E. A. Lee and D. G. Messerschmitt, Digital Communications. Kluwer Academic Publishers, 2nd ed., 1994.

[12] A. A. M. Saleh and R. A. Valenzuela, "A statistical model for indoor multipath propagation," IEEE J. Select. Areas Commun., vol. 5, pp. 128 137, February 1987.

[13] S. Haykin, Adaptive Filter Theory. Prentice Hall Information and System Sciences Series, Prentice Hall, 3rd ed., 1996.

[14] A. Gersho and R. M. Gray, Vector Quantization and Signal Compression. Kluwer Academic Publishers, 1991.

[15] J. G. Proakis, Digital Communications. WCB McGraw-Hill, 3rd ed., 1995. 